\title{
A review of the eco-industrial park evaluation of high energy consumption and high pollution industry
}

\author{
Jun $\mathrm{Liu}^{1}$, Yang Wang ${ }^{2}$, Hongjing Zhang ${ }^{1}$, Jieping Han $^{1}$, Jindong Cui \\ ${ }^{1}$ School of Economics \& Management, Northeast Dianli University, Jilin City, Jilin 132012, China \\ ${ }^{2}$ Human Resources Department, Shiyan Power Supply Company, STATE GRID Corporation of \\ China, Shiyan City, Hubei, 442000, China
}

527788089@qq.com

Keywords: energy consumption and high pollution industry; eco-industrial park; environmental benefit, circular economy

\begin{abstract}
At present, haze weather occurs in each big city of China in different extent. The problem of haze has become one of the biggest environmental problems now and the high energy consumption and high pollution industry is the source of haze weather. This paper takes the evaluation of eco-industrial park as the breakthrough point, taking research of high energy consumption and high pollution industries in the main body of the study and putting forward the direction of development about the high energy consumption and high pollution industry in eco-industrial park according to the problem of the research.
\end{abstract}

\section{Introduction}

Eco-industrial park is a new form of industrial organization that takes one industry as the main body and with a plurality of the coordinated development of industry, which is based on the principle of circular economy and industrial ecology. It is of the most green concept, economic benefits, environmental protection significance. In China, the eco-industrial park development is relatively slow. Until the first half of 2014, more than 80 parks were approved to construct, however only 23 parks of them were considered acceptable and were named as the national ecoindustrial park. They are mainly related to the chemical industry, iron and steel, sugar, thermal power, metal smelting, petroleum coke and other high energy consumption and high pollution industry. Lei Ming, Zhong Shuhua[1] selected the literature since 2003 to 2007, overviewed the eco-industrial park from three aspects of principles, methods and index. They found that the evaluation of index is more complicated and pertinence is absent. Effective evaluation is beneficial to the continuous improvement of Eco-industrial Park, so as to improve the economic benefits, environmental benefits, social benefits. This paper summarized the breakthrough point and the evaluation of the park of high energy consumption and high pollution of the park, and find the existing problems, hoping to contribute to the study of high energy consuming industry eco-industrial park.

\section{The method of evaluation}

For any question, we should choose a suitable starting point, in order to make the problem clearer, and make the study more reasonable. According to the evaluation about the ecological industrial park, different scholars have carried out the evaluation from different angles.

Symbiotic efficiency is equal to symbiotic benefits divided by symbiotic cost. Gan Yonghui, Yang Jiesheng, Huang Xinjian[2] analysed, forecast, assessed the running benefit of eco-industrial park from above perspective; Eco-industrial park is composed of a plurality of industry and different enterprise. In a sense, eco-industrial park can be regarded as a symbiosis network. According to the characteristics of Ecological Symbiosis Network Industrial Park, Yu Zheng[3]etc proposed a node importance evaluation method to evaluate the eco-industrial park. 
The environment is one of the most important evaluation factors in the ecological industrial park. Rui Junwei, Zhou Beibei, Qian Yi, Sun Jing[4]believes that environmental impact is difficult to directly add, so it is essential to find measurement. The study found that we can account environmental impact from two perspectives of ecological efficiency and ecological footprint, then construct a model to evaluate the ecological efficiency of ecological industrial park, which is composed of environmental pollution, ecological resources demand and energy account; Hung-Suck Park, Shishir Kumar Behera[5] think that the ecological efficiency includes an economic indicator and three environmental indicators, which take industrial symbiosis network as evaluation. Using data of 2007-2012, they found that ecological efficiency is enhanced by $10 \%$ among 21 enterprises in seven symbiotic network. The eco efficiency indicators based on the ecological symbiosis network is conducive to a better direction for the ecological Industrial Park; Liu Wei, Tian Jinping, Li Xing[6] evaluated the eco efficiency of eco-industrial park using DEA method from the angle of environmental performance and environmental management. According to the new standard of the State Environmental Protection Administration, Raymond P. Cote, Tsuyoshi Fujita[7] etc found that criteria evaluated the eco-industrial park mainly from the ecological efficiency. They verified the feasibility and applicability of this index, putting forward a valuable advice for improving ecological index system. Xiong Hongbin, Wang Yufang, Xia Xiaoyu[8] analyzed and evaluated the eco-industrial park from a perspective of low carbon development, evaluating the environment benefit of Hefei high tech zone industrial park using slack variables measurement model, drawing a conclusion that the $\mathrm{CO}_{2}$ emission of unit of industrial added value was negatively correlated with and environmental efficiency, promoting the study of ecological benefit.

From energy recovery angle of dry sludge and oil products, Qiang Liu, Peipei Jiang, Jun Zhao, Bo Zhang[9] taken up research into the life cycle of industrial symbiosis in eco-industrial park.They found this method can make the waste and energy management integration and can reduce waste, the total energy consumption and operating costs. This method can establish index system concluding global warming potential evaluation system value, acidification potential, eutrophication potential, harmful gas, the total environmental impact of human potential. Replacement rate of the sludge drying and refining oil taking the place of fossil fuel is $14 \%$.The mixture burning of the dry sludge, finished product oil and coal will emit less carbon dioxide, hydrogen, nitrogen, nitrous oxide and carbon monoxide, and will facilitate the development of environment. Based on the circulation economy theory and symbiosis theory, Zhang Yongcheng[10] think that the most important basis for evaluation of eco-industrial parks is the utilization of resources and reduction of waste emissions, so the construction of waste optimal emission measure model is essential. That model can achieve the minimum of waste discharge and provides the optimal mode for the operation of circular economy system in eco-industrial park.

According to fundamental theory of dissipative structure, the "entropy" in thermodynamics is introduced to the evaluation methods by $\mathrm{Li}$ Ren[11]. He analyzed the ordering degree and development trend of eco-industrial park and built management system of sustainable development which is good for eco-industrial park, based on the evaluation results.

From the angle of the terrain, Wang Qinqin, Jiang Ying, Zhao Guoqing[12] established multi-level rating system by using AHP and multi factor evaluation method and analyzed the project base data. That came to the conclusion that the ecological land and the legitimate rights of farmers should be paid more attention in the early of eco-industrial park.

From the angle of micro, meso and macro, Li Di,Hu Yongjun and Xiao Zhongliang[13] assessed ecological level of Changsha Zhuzhou Xiangtan experimentation area and put forward valuable opinions according to establishment, cleaner production, circular economy.

From internal, external members, irresistible factors and structural relationship, Su Qingfu, Zhao tao[14] filtered and evaluated many risk scenarios from the angle preliminary, dual and multiple standard, using quantitative rating and mutual dependence analysis to establish hierarchical holographic model, which was used to analyze eco-industrial park, and then found the main risk. That method is good for decision-makers to seize the key issue and prevent the risk. 
Dominance is the internal driving force for the development of eco-industrial parks. Adjustment degree is the response to external environmental action and self maintenance ability. Cycle degree is the dynamic balanced system trend caused by the internal. From above three angle, Shang hua[15] constructed the network dimension framework and evaluated Dalian eco-industrial park by collected data, putting forward constructive suggestions.

From the energy theory, Zhang Yun, Chen Xiuqiong, Wang Tongyao[16] unified logistics, energy flow, currency flow for the energy unit. They applied this method to iron and steel industrial park and analyzed ecological efficiency, the structure and function of the park, evaluating the level of sustainable development. That evaluation reflected economic benefit and ecological benefit, being good for valuable suggestions proposed. From the angle of the utilization of resources and waste emission, M. M. Jiang, J. B. Zhou, G. O. Chen set ecological efficiency, resources use efficiency and environmental emissions intensity to assess the coal-fired power plant.

Eco-industrial park is a series of related enterprises community pursuing the benefit which could be called symbiosis. It constitutes a whole from economy, environment, resources, energy and social, seeking the maximum of the overall benefits. All authors seized the characteristics to analyze and evaluate the eco-industrial park from the angle of symbiotic, environment, ecology, structure, distribution and utilization of resources, sustainable development and material circulation. Facing emerging issues of various aspects, authors put forward the reasonable suggestions and promoted the development of eco-industrial park. At the same time, it is not difficult to find that evaluation is only involved economy, environment, resource, energy, while aiming at social evaluation is relatively rare. Perhaps that angle will become the future focus. Setting indicators and selecting data are difficult, needing more sociological experts participate the research.

\section{Evaluation of high energy consumption and high pollution industry}

The establishment of eco-industrial park is to pursuit the maximum of economic, social and environmental benefit. Those high energy consumption and high pollution industries, for example, chemical, steel, sugar, power thermal, metal smelting, petroleum coke, as pillar industry should enjoy a set of special evaluation method and system according to their own characteristics.

In the electric thermal, Qiang Liu, Peipei Jiang, Jun Zhao[9] collected waste heat and steam coming from the central heat supply companies to dry sludge and refine oil products. These dried sludge and refined oil can become the substitute of fossil fuel. The research result showed that the replacement rate of sludge, coal was $14 \%$ and industrial symbiosis had the minimum impact on the environment. The thought that recycling energy from the heating company made waste and energy management integration is beneficial to save the cost and make waste profitable. Aiming at resource utilization, system emission and life cycle of coal-fired power plant, M. M. Jiang, J. B. Zhou, G. O. Chen[17] compared average model of emission and operation efficiency, new source performance standards model and low emission boiler system model. This paper puts forward the effective evaluation system for the coal-fired power plant.

In the chemical area, Chen Yu, Liu Suling, Zhang Shushen, Zhang Yun, Cheng Lei and Wang Huanlei[18] established evaluation system of sustainable development containing criteria layer and field layer based on chemical eco-industrial park features. The criteria layer includes the current development situation, coordination degree, potential; Field layer includes 36 indicators ranging from economic conditions, environmental conditions, environmental management, ecological network to the technical and conscious level. Jinping Tian, Han Shi, Ying Chen, Lujun Chen[19] applied material flow analysis to the evaluation process of chemical eco-industrial park. Though analyzing the conversion efficiency of sulfur, they made the conclusion that calcium sulfate waste is one of the main source in the chemical industry park crisis and the end process dealing with calcium sulfate waste will become a hot issue in terms of chemical industrial park.

In the iron and steel, Zhang Yun, Chen Xiuqiong, Wang Tongyao, Chen Yu, Liu Suling, Zhang Shushen[16] applied energy theory to analyze ecological efficiency and structure functional features and evaluated the level of sustainable development. The system can fully reflect the ecological and economic benefits. 
The above scholars put forward the corresponding evaluation system, verifying and proposing some future problems, according to the respective features of the thermal power, chemical, iron and steel. America energy department recommended to establish energy region in some previous nuclear address, which is energy based eco-industrial park[20].That park will strengthen the renewable energy, nuclear, coal, waste management and energy transfer technology, converging a large number of high energy consumption and high pollution industries. After 25 years of development, the environment will be greatly improved. Energy eco-industrial park will become the research trend in the future.

\section{The existing problems and the prospects}

With the rapid development of economy, the importance of the environment is more and more valued by people and eco-industrial park has become one of the best ways to solve the issues of resources, economy, environment. With the continuous establishment of eco-industrial park, scholars pay more and more attention to its evaluation. Evaluation is beneficial to perfect the development of eco-industrial park, to propose rational references, and to meet the strategic requirements of circular economy and sustainable development.

The scholars' research should connect the evaluation method with the organic operation of eco-industrial park real. It has the certain instruction function to the actual. Comparing with the previous research, indexes designed, more unique, targeted, relatively small, can research different park and simply applied.

The present study focused on the resources, economy, environment, while more and more attention should be paid to human factors. From the perspective of society, setting evaluating indexes which are easily collected will become the future research direction. On this basement, constructing index system of resource, economy, environment, society targeted will be beneficial to the long-term development of high energy consumption and high pollution eco-industrial park (Fig.1).

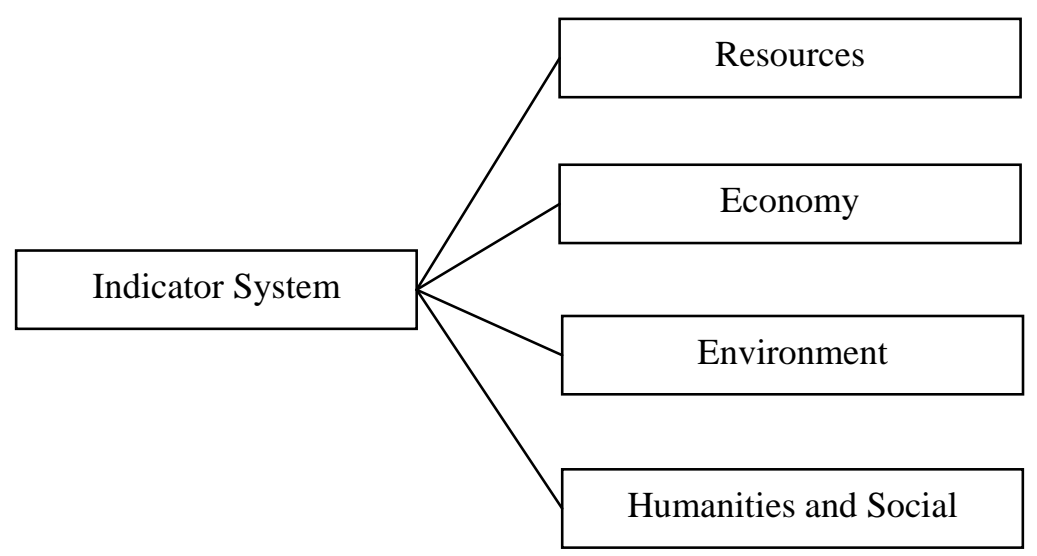

Fig.1. The experimental results

At present, the constructions of high energy consumption and high pollution eco-industrial park are divisional. If gathering a lot of high energy consumption and high pollution industries and establishing energy eco-industrial park in the nuclear energy address, using renewable energy, nuclear, coal and waste management and energy transfer technology, may reduce the pollution area of the environment and may make resources used efficiently. It may become the future development direction of the ecological industry park (Fig.2). 


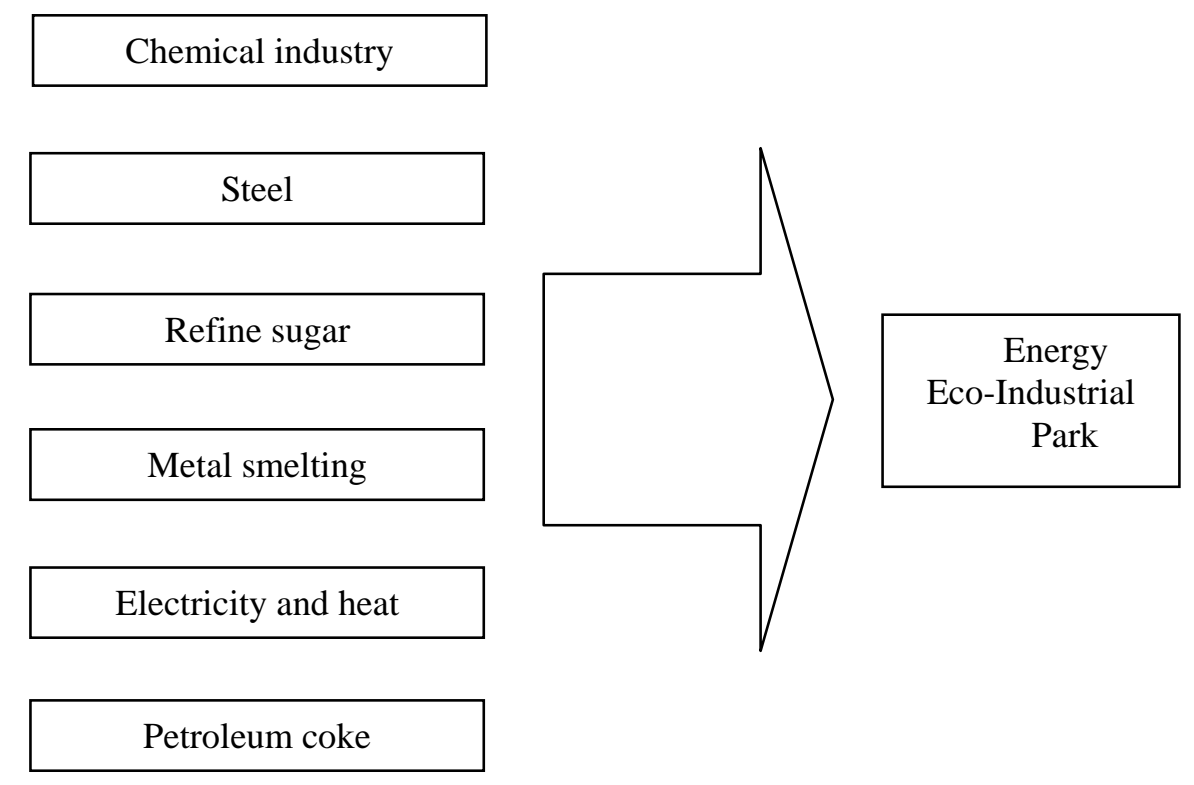

Fig.2 Energy Eco-Industrial Park

\section{Acknowledgement}

In this paper, the research was sponsored by the ministry of education for humanities and social science research fund(12YJA630035) and philosophy and social science fund project of Jilin province(2014B211, 2014B212, 2014B213, 2013BS75, 2013BS76).

\section{References}

[1] Lei Ming, Zhong Shuhua. Review on the Eco-Industrial Parks Evaluation[J]. Science \& Technology Progress and Policy, 2010, 27(6): 156-160.

[2] Gan Yonghui, YANG Jiesheng, HUANG Xinjian. Study on Industrial Symbiosis Efficiency in Eco-Industrial Parks[J]. Journal of Nanchang University, 2008, 39(3): 75-80.

[3] Zeng Yu, Xiao Renbin, Li Xiangmei, et al. A Resilience Approach to Symbiosis Networks of Ecoindustrial Parks Based on Cascading Failure Model[J]. Mathematical Problems in Engineering, 2013, 2013: 1-11.

[4] Rui Junwei, Zhou Beibei, Qian Yi, et al. Research and Application of Method for Evaluation of Eco-Efficiency of Eco-Industrial Parks(EIP)[J]. Journal of Ecology and Rural Environment, 2013, 29(4): 466-470.

[5] Park Hung Suck, Behera Shishir Kumar. Methodological aspects of applying eco-efficiency indicators to industrial symbiosis networks[J]. Journal of Cleaner Production, 2014, 64: 478-485.

[6] Liu Wei, Tian Jinpin, Li Xiang, et al. Eco-efficiency Assessment of Eco-industrial Parks in China with Date Envelopment Analysis[J]. China Population, Resources and Environment, 2012, 22(5): 93-97.

[7] Geng Yong, Zhang Pan, Raymond P, et al. Assessment of the National Eco-Industrial Park Standard for Promoting Industrial Symbiosis in China[J]. Journal of Industrial Ecology, 2009, 13(1): 15-26.

[8] Xiong Hongbin, Wang Yufang, Xia Xiaoyu, et al. Environmental efficiency evaluation of eco-industrial parks under low carbon development pathway based on SBM model[J]. Environmental Pollution \& Control, 2013, 35(7): 95-100. 
[9] Liu Qiang, Jiang Peipei, Zhao Jun, et al. Life cycle assessment of an industrial symbiosis based on energy recovery from dried sludge and used oil[J]. Journal of Cleaner Production, 2011, 19(15): 1700-1708.

[10] Zhang Yongcheng, Wang Chengzhang. The measure method of ecological industrial park resource use and waste emissions[J]. Statistics and Decision, 2009 (18): 15-18.

[11] Li Ren, Yao Shumei, Shan Shan, et al. Eco-industrial park assessment and management based on dissipative structure theory[J]. Environmental Engineering, 2009, 1: 480-483.

[12] Wang Qinqin, Jiang Ying, Zhao Guoqing, et al. Method and application of land use suitability evaluation based on GIS: Singapore Huaihua industrial park example[J]. Planners, 2011, 27(4): 52-56.

[13] Li di, Hu Yongjun, Xiao Zhongliang, et al. On eco-friendly growth of industries in Changsha-Zhuzhou-Xiangtan area[J]. China Openning Herald, 2010(1): 101-105.

[14] Su Qingfu, Zhao Tao. Risk identification and filter research of eco-industrial park. Statistics and Decision, 2012 (1): 76-79.

[15] Shang Hua. The stability evaluation of eco-industrial parks[J]. Science Research Management, 2012, 33(12): 142-148.

[16] Zhang Yun, Chen Xiuqiong, Wang Tongyao, et al. Evaluation of the sustainability of iron and steel eco-industrial parks based on emergy theory[J]. Journal of Hunan University(Natural Sciences), 2010, 37(11): 66-71.

[17] Jiang M M, Zhou J B, Chen G Q. Unified process assessment for resources use and waste emissions by coal-fired power generation[J]. Communications in Nonlinear Science and Numerical Simulation, 2010, 15(9): 2723-2733.

[18] Chen Yu, Liu Suling, Zhang Shushen, et al. Evaluation of sustainable development in chemical eco-industrial park[J]. Modern Chemical Industry, 2010 (12): 86-90.

[19] Tian Jinping, Shi Han, Chen Ying, et al. Assessment of industrial metabolisms of sulfur in a Chinese fine chemical industrial park[J]. Journal of Cleaner Production, 2012, 32: 262-272.

[20] Greenberg Michael R. Energy parks for former nuclear weapons sites? Public preferences at six regional locations and the United States as a whole[J]. Energy Policy, 2010, 38(9): 5098-5107. 